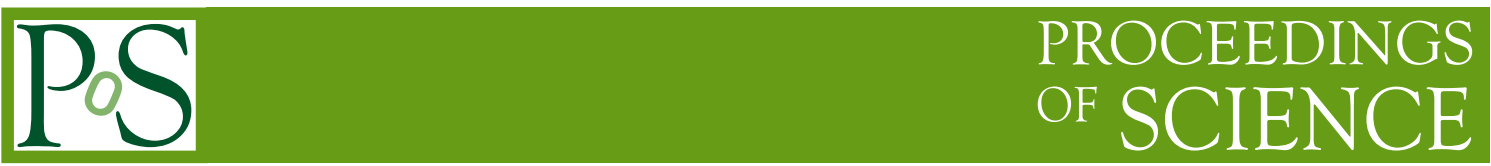

\title{
Heavy Flavor Physics with the ATLAS Detector
}

\author{
Charilaos Tsarouchas* \\ CERN \\ E-mail: tsarouchecern.ch
}

This note presents a big range of flavor physics results with the ATLAS detector based on LHC data collected in $p p$ collisions between 2010 and 2012. This includes measurements of open charm and beauty mesons and quarkonia production. The agreement of these first results with Monte Carlo expectations and PDG world averages demonstrate a good detector performance. Furthermore, in the higher integrated luminosity phase, results include precision measurements in the flavor sector which allow indirect searches for New Physics.

Proceedings of the Corfu Summer Institute 2012 "School and Workshops on Elementary Particle Physics and Gravity"

September 8-27, 2012

Corfu, Greece

${ }^{*}$ Speaker. 


\section{The ATLAS Detector}

The ATLAS experiment [36] is a multipurpose particle physics detector of $44 \mathrm{~m}$ in length, $25 \mathrm{~m}$ in diameter divided into three main regions, a central region called barrel and two endcap regions, one on each side. The very center of the detector, where the two proton-beams of LHC collide, is called the Interaction Point (IP). Moving outward from the interaction point, the detector consists of a tracking system able to measure the directions and momenta of the charged particles. The calorimeter system measures the energies of the electrons, photons and hadrons. The muon spectrometer measures the momentum and position of the muons that have enough energy to reach it. Furthermore, a magnet system provides a magnetic field, allowing the tracker and the muon spectrometer to perform measurements of the momentum of charged particles. For the flavor physics studies it is of primary importance that the tracking detector and the muon spectrometer are operating correctly and the relevant particles are reconstructed only when strict quality selection criteria are satisfied and only when the LHC beams are declared to be stable.

\section{Open Charm, Open Beauty and Baryons}

We declare the open beauty/charm mesons the mesons of a $c / b$ quark together with another quark that is not the $\bar{c} / \bar{b}$ (so to distinguish from quarkonia). The open charm mesons, due to their large production cross sections and clean experimental signatures are among the first hard QCD processes to be measured at LHC (examples in fig.1). The charm mesons $D^{*+-}, D^{+-}, D_{s}^{+-}$have been observed with the ATLAS detector and their productions has been measured in pp collisions at $\sqrt{s}=7 \mathrm{TeV}$ using an integrated luminosity of $1.1 n b^{-1}$ [1]. The differential cross sections $d \sigma / d p_{T}$ and $d \sigma / d|\eta|$ were calculated for $D^{* \pm}$ and $D^{ \pm}$production and the corresponding NLO QCD predictions within the large theoretical uncertainties. Charged $B$ mesons are clearly observed and reconstructed exclusively in the ATLAS detector through their decay to $J / \psi K^{ \pm}$final state [2]. Their fit to the peak yields a central value of $5283.2 \pm 2.5$ (stat.) $\mathrm{MeV}$, compatible with the world average. The mesons $B_{d}^{0}, B_{s}^{0}$ are observed in ATLAS with a fitted mass of $5279.6 \pm 0.9$ (stat) MeV and $5364.0 \pm 1.4$ (stat) $\mathrm{MeV}$ respectively, consistent with the world average values within their statistical uncertainties [3]. Furthermore, in a subsequent study [4] the $B_{d}^{0}$ and $B_{s}^{0}$ lifetimes are calculated through a simultaneous mass-lifetime fit and found to be in accordance with the world average values. The $B_{c}^{+}$and the $B_{c}^{-}$are the mesons of the two heaviest quarks that can make a stable state and are studied by the ATLAS detector too [5]. The fitted mass measured, 5620.6 \pm 1.6 (stat.) $\mathrm{MeV}$, is consistent with the world average. Finally, apart from the $B$ meson studies, there are baryon studies which raise high physics motivation. For instance, the $\Lambda_{b}$ baryon can not be produced at $B$-factories (which are tuned to produce $B$ meson pairs) and thus can be studied in hadron colliders. Nevertheless, measurements of the CDF and D0 in Tevatron show differences[6]. ATLAS studies of mass and lifetime of the $\Lambda$ baryon in the decay channel $\Lambda_{b} \rightarrow J / \psi\left(\mu^{+} \mu^{-}\right) \Lambda^{0}\left(p \pi^{-}\right)$[7] agree with the world average values and with the recent LHCb results concerning the $\Lambda_{b}$ mass [8].

\section{Quarkonia}

Quarkonium is a meson that is composed of a quark and an antiquark. Typical quarkonia are the bound states of the c quark and its antiquark, the $\psi$ states (charmonium) and the b quark and 
its antiquark, the $\Upsilon$ states (bottomonium). Although the first quarkonia were observed in seventies $(J / \psi$ observation [9]), still there is not clear understanding of the quarkonium production mechanisms which explain both cross sections and spin alignment. Studies of such heavy flavor states provide a good ground for testing the validity of quantum chromodynamics (QCD). The conclusions derived from the initial $J / \psi$ observations in the ATLAS detector [10] include agreement of the fitted mean value $3.095 \pm 0.004 \mathrm{GeV}$ and agreement of the mass resolution as a function of the muon pseudorapidity with Monte Carlo expectations (see fig. 2). Subsequent relevant studies of higher integrated luminosity, permit measurements of the differential cross sections inclusive, prompt and non prompt $J / \psi$ production in $p p$ collisions [11]. It should be mentioned that in this study the systematic uncertainties of $J / \psi$ inclusive production cross section include the correction of the number of $J / \psi$ for the kinematic acceptances which depend on the (not known in LHC) $J / \psi$ spin alignment. The prompt $J / \psi$ are produced directly very close to the primary vertex of the $p p$ collisions while other ones non-prompt originate from $B$-hadron decays and have a measurably displaced decay point due to the lifetime of the parent particle. Experimentally, it is possible to distinguish between these two decays based on the pseudo proper lifetime $\tau=L_{x y} m_{J / \psi}^{P D G} / p_{T}^{J / \psi}$, where $L_{x y}$ is the vertex distance between the primary and the $J / \psi$ vertex projected onto the transverse momentum direction, $m_{J / \psi}^{P D G}$ is the world average value for the $J / \psi$ mass and $p_{T}^{J / \psi}$ is the transverse momentum of the $J / \psi$. The total production cross section for non - prompt $J / \psi$ is compared to Fixed Order Next - to - Leading Logarithm (FONLL) calculations [12] show good agreement. The total integrated prompt $J / \psi$ production cross section is compared with predictions of the Color Evaporative Model [13][14] which agree relatively well in scale but do not agree well in shape, with predictions of the Color Singlet Model (CSM) at Next to Leading Order (NLO) [15] which agree relatively well in shape but not well scale and with predictions of the CSM at partial Next to
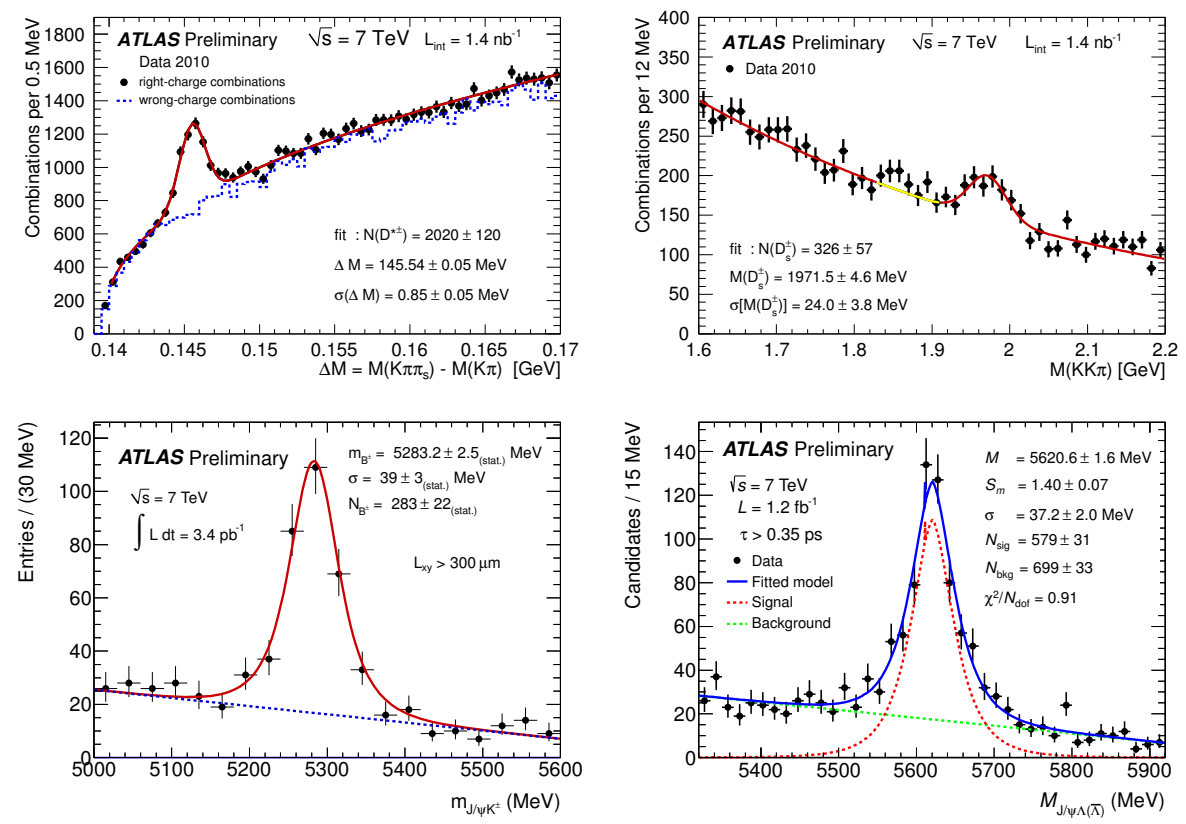

Figure 1: Observations of the $D^{* \pm}$ (top-left), $D_{s}^{ \pm}$(top-right), $B \pm$ (bottom-left) $\Lambda_{b}$ (bottom-right) 
Next to Leading Order (NNLO) [16] which agree relatively well both in shape and scale.

Similarilly to the charmonium, the bottomonium resonances are discovered in the 1970s [17], but still until today there is no coherent theoretical picture for $\Upsilon$ hadroproduction. Moreover, there are significant disagreements of past experimental results between the two CDF and D0 in Tevatron [18] [19] [20]. Results of the measurement of the $\Upsilon(1 S)$ production cross section in $p p$ collisions from ATLAS [21] intend to shed further light on this puzzle. The cross-section measurement is compared with theoretical predictions of CSM (NLO) which do not agree while reasonable agreement is observed with NRQCD predictions (see fig. 3).

Finally, the rich quarkonium physics program of the ATLAS detector includes the searches of the $\chi_{b}(3 P)$ state which was predicted to exist just below the $B-B$ threshold and observed for a first time at a hadron collider. The reconstruction of this state is done through the radiative decay $\chi_{b}(n P) \rightarrow Y(1 S) \gamma$ and $\chi_{b}(n P) \rightarrow Y(2 S) \gamma$ in which the $Y$ states are decaying to two muons and the
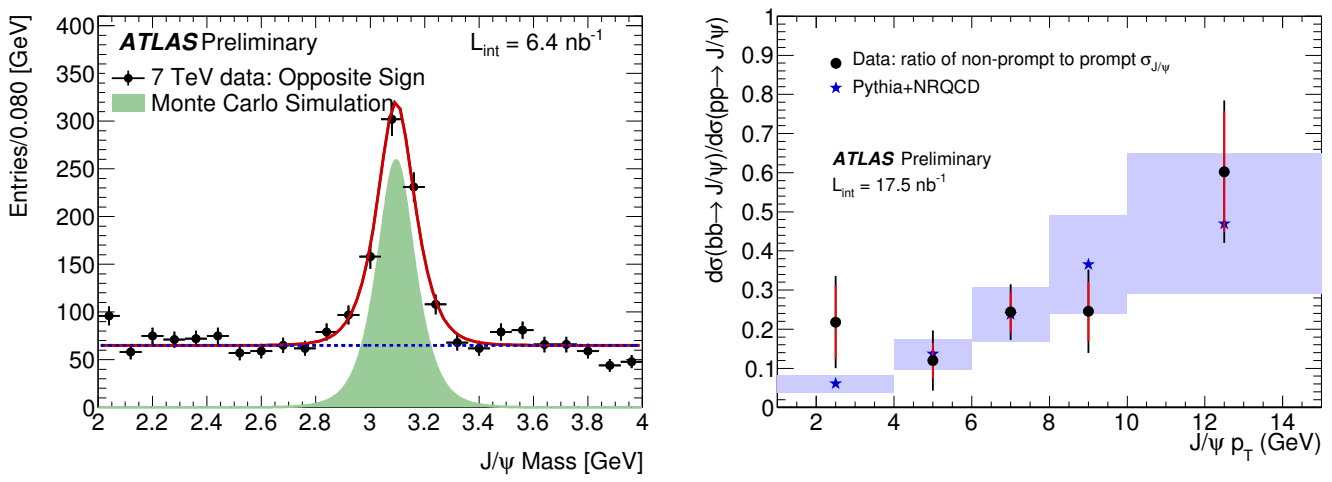

Figure 2: In the invariant mass distribution of reconstructed $J / \psi$ candidates (left), the date are represented with the points with error bars while the solid area represents prompt $J / \psi$ coming from MC. The ratio of the non-prompt to prompt $J / \psi$ cross sections as a function of $J / \psi_{p^{T}}$ (right). The overlaid band represents the MC prediction.
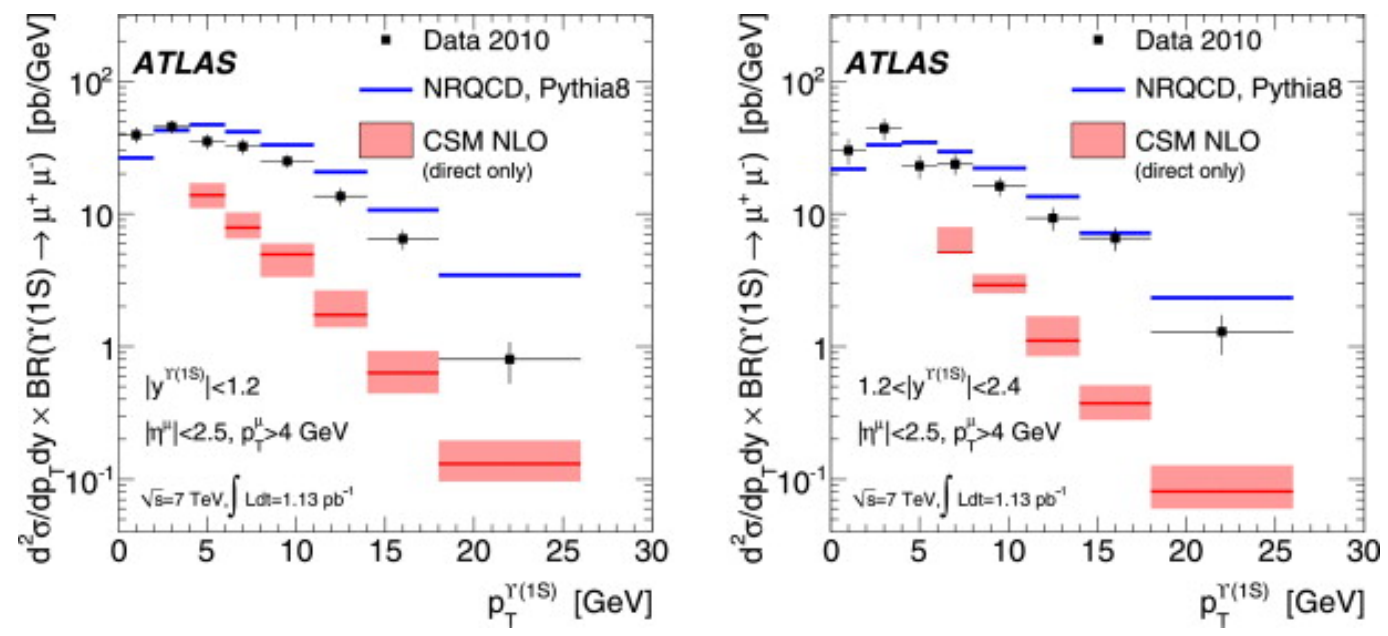

Figure 3: The differential $\Upsilon(1 S)$ cross section as a function of $\Upsilon_{p^{T}}$ for the central (left) and forward (right) regions together with CSM NLO and NRQCD predictions. 
photon is reconstructed either through $e^{+} e^{-}$conversion or direct calorimetric measurement[22]. The new structure which is centered at a mass of $10.530 \pm 0.005$ (stat) \pm 0.009 (syst) GeV is in reasonable agreement with the theoretical predictions [23].

\section{Heavy Flavor Probes of New Physics}

Effects of physics beyond the Standard Model (SM) can be probed by measurements in the flavor sector. The purely leptonic rare decay $B_{S} \rightarrow \mu^{+} \mu^{-}$is forbidden at tree level in the SM as Flavor Changing Neutral Current (FCNC) process and instead is dominated by a $Z$ boson penguin diagram and a box diagram with the exchange of two $W$. The really small calculated rates of FCNC processes together with the relatively simple theoretical description and the very clean experimental signature establish the rare $B$ decays as an excellent benchmark channel for probing effects beyond the SM. Furthermore the origin of $C P$ violation remains one of the most important questions and NP phenomena may alter $C P$ violation in $B$-decays. The measurement of the $B_{s}$ mixing phase, responsible for $C P$ violation in the decay $B_{s}^{0} \rightarrow J / \psi \phi$, is predicted to be small within $\operatorname{SM}\left(O\left(10^{-2}\right)\right)$ and any measured excess would be an indication of physics beyond the SM.

\subsection{Rare Decays $B_{s} \rightarrow \mu^{+} \mu^{-}$}

A first search of the rare decay $B_{s} \rightarrow \mu^{+} \mu^{-}$is performed in ATLAS using $L_{\text {int }}=0.04 \mathrm{fb}^{-1}$ [24] where events are triggered with a di-muon trigger which required a $p_{\mathrm{T}}>4$ for each muon. The $\operatorname{Br}\left(B_{s}^{0} \rightarrow \mu^{+} \mu^{-}\right)$limit is calculated through the following formula

$$
\operatorname{Br}\left(B_{s}^{0} \rightarrow \mu^{+} \mu^{-}\right)=N_{\text {obs }} \text { SES }
$$

where $N_{\mathrm{obs}}$ is the number of observed events. The SES is the Single Event Sensitivity, given by

$$
\mathrm{SES}=\mathrm{Br}_{\mathrm{ref}}\left(B^{ \pm} \rightarrow J / \psi K^{ \pm}\right) \frac{f_{u}}{f_{s}} \frac{1}{N_{\text {ref }}} \frac{A_{\text {ref }} \varepsilon_{\text {ref }}}{A \varepsilon}
$$

where $\mathrm{Br}_{\text {ref }}\left(B^{ \pm} \rightarrow J / \psi K^{ \pm}\right), N_{\text {ref }}, A_{\text {ref }}$ and $\varepsilon_{\text {ref }}$ are the branching ratio of the abundant reference channel $B^{ \pm} \rightarrow J / \psi K^{ \pm}$, the number of $B^{ \pm}$candidates, the geometrical acceptance of the detector, the (reconstruction and trigger) efficiency respectively, while $A$ and $\varepsilon$ are the geometrical acceptance of the detector and the (reconstruction and trigger) efficiency, respectively. In addition, $f_{u} / f_{s}=$ $0.267 \pm 0.021[25]$ is the ratio of the fragmentation functions of the $u$ and $s$ quarks. The analysis that is performed is blind so that the signal region $[5066,5666] \mathrm{MeV}$ is not revealed until the limit extraction method is completely defined. The data are divided into three subsets, according to the three mass resolution families (barrel, transition and end-cap) so to maximally exploit the data information. In order to avoid biases, the odd-numbered side-band events are used in signal/background comparisons, multivariate classifier training and selection optimization, while the even-number events are exclusively used for the final background interpolation and the extraction of the $B^{ \pm}$yield. The background of the signal can be split into two main parts, a non-resonant $\left(B \rightarrow \mu^{+} \mu^{-} X\right)$ continuum background and a resonant part (details can be found in appendix $\mathrm{J}$ of [28]). Concerning the signal-background discrimination, 14 variables with the highest separation power, uncorrelated to $B_{s}$ mass, were chosen as inputs for a multi-variate analysis which uses a 
Boosted Decision Trees (BDT) within the TMVA package [27]. Finally, A Confidence Level (CLs) [26] statistical extraction method is used to set an upper limit of the branching ratio (see pic. 4). The expected limit is $2.3+1.0 \times 108$ at $95 \% \mathrm{CL}$ and the observed limit is $2.2 \times 108$ at $95 \% \mathrm{CL}$, compatible with the expected background plus a SM signal within $1 \sigma\left(1 C L_{s}+b=84 \%\right)$. The above result is used in the calculation of the combined ATLAS, CMS, LHCb limit [29] which is the best existing limit at the time this note is written and it saws no significant NP enhancement of the branching ratio with respect to the SM.

\subsection{Measurement of $B_{s} \rightarrow J / \psi \phi$}

The $B_{s}$ meson is a quantum superposition of the $B_{s}^{H}$, heavy and $B_{s}^{L}$, light (of width $\Delta \Gamma_{s}$ ) mass eigenstates which deviate from the $C P$ eigenstates described within the SM by the $C P$ violating phase $\phi_{s}$. This phase is related to CKM quark mixing matrix elements via the relation $\phi_{s} \simeq 2 \beta_{s}$ and is calculated to be small, $\phi_{s}=0.03680 .0018$ [31]. There are models which predict that NP will change the expected values of $\phi_{s}$ and $\Delta \Gamma_{s}$ significantly [32] [33] and the decay $B_{s} \rightarrow J / \psi \phi$ can be used to test such predictions. The $B_{s}$ is a pseudoscalar meson while the final state is composed by two vector mesons $(J / \psi$ and $\phi)$ which can have an even or odd relative orbital momentum $\mathrm{L}$ in the final states. The final states, due to total angular momentum conservation, is an admixture of $\mathrm{CP}$-even and CP-odd states and can be separated statistically through the time-dependence of the decay and angular correlations amongst the final-state particles.

The analysis in ATLAS is preformed using $4.9 \mathrm{fb}^{-1}$ of integrated luminosity of $p p$ collisions at a center of mass energy $\sqrt{s}=7 \mathrm{TeV}$ [30]. A measurement of the decay parameters including the $\phi_{s}$, $\Delta \Gamma_{s}, \Gamma_{s}$ and the transversity amplitudes is done via a unbinned maximum likelihood 26 parameters simultaneous fit to the distributions of mass, proper time and angular coordinates.

$$
\ln L=\sum_{i=1}^{N}\left[w_{i} \cdot \ln \left(f_{s} F_{S}\left(m_{i} t_{i} \Omega_{i}\right)+f_{s} f_{B^{0}} F_{B^{0}}\left(m_{i} t_{i} \Omega_{i}\right)+\left(1-f_{s} \cdot\left(1+f_{B^{0}}\right)\right) F_{b k g}\left(m_{i} t_{i} \Omega_{i}\right)\right)\right]+\ln P\left(\delta_{\perp}\right)
$$

where $\mathrm{N}$ is the number of selected candidates, $F_{S}, F_{B}^{0}$ and $F_{b} k g$ are the probability density functions

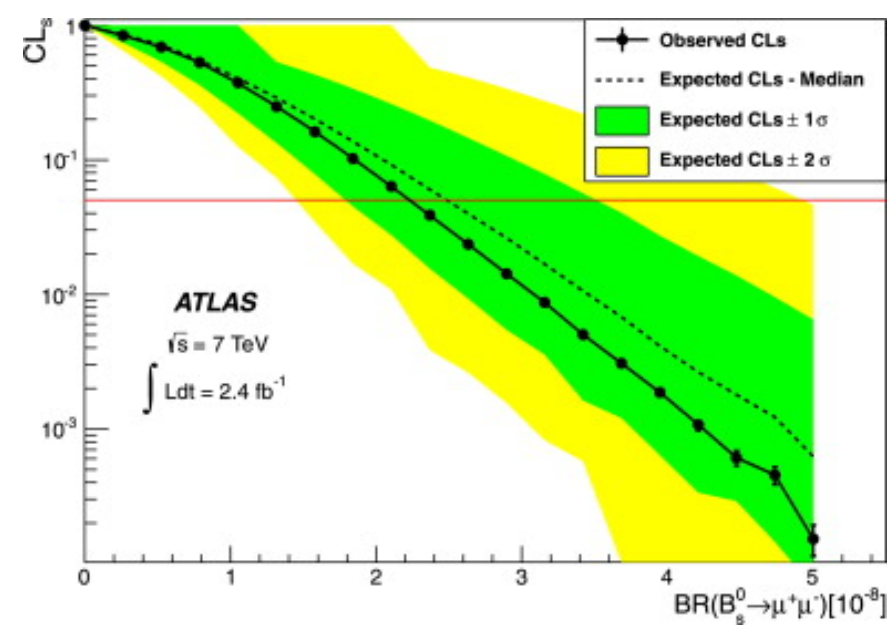

Figure 4: Observed CLs (circles) as a function of $\operatorname{Br}\left(B_{s} \rightarrow \mu \mu\right)$. The expectation values are represented by the dashed line while the green and yellow bands represent the $\pm 1 \sigma$ and $\pm 2 \sigma$ fluctuations. The $95 \% \mathrm{CL}$ limit is indicated by the horizontal (red) line. 
modeling the signal, the specific $B^{0}$ background and the other background distributions, $w_{i}$ is a weighting factor to account for the trigger efficiency, $f_{s}$ is the fraction of signal candidates, $f_{B^{0}}$ is the fraction of peaking $B^{0}$ meson background events relative to the number of signal events, $m_{i}$ is the mass, $t_{i}$ is the proper decay time and $\Omega_{i}$ the decay angles measured from the data for each event i respectively. $P\left(\delta_{\perp}\right)$ is a constraint on the strong phase.

The likelihood contour fits (see fig. 5) of the $\Delta \Gamma_{s}, \phi_{s}$ parameters reveal consistency with the SM predictions and similar measurement precision to the Tevatron $\left(\phi_{s}\right)$ [34] and $\operatorname{LHCb}\left(\Delta \Gamma_{s}\right)[35]$ results.

\section{References}

[1] ATLAS Collaboration, Measurement of $D^{(*)}$ meson production cross sections in pp collisions at sqrts $=7 \mathrm{TeV}$ with the ATLAS detector, ATLAS NoteATLAS-CONF-2011-017.

[2] ATLAS Collaboration, Observation of the $B^{ \pm}$meson in the decay $B^{ \pm} \rightarrow J / \psi\left(\mu^{-}+\mu\right) K^{ \pm}$in ATLAS. ATLAS NoteATLAS-CONF-2011-098.

[3] ATLAS Collaboration, Observation of the $B_{d}^{0}$ and $B_{s}^{0}$ mesons in the decay $B_{d}^{0} \rightarrow J / \psi K^{* 0}$ and $B_{s} \rightarrow J / \psi \phi$ in ATLAS, ATLAS Note ATLAS-CONF-2011-050.

[4] ATLAS Collaboration, Measurement of the $B_{d}^{0}$ and $B_{s}^{0}$ lifetimes in the decay $B_{d}^{0} \rightarrow J / \psi K^{* 0}$ and $B_{s} \rightarrow J / \psi \phi$ in ATLAS, ATLAS Note ATLAS-CONF-2011-092.

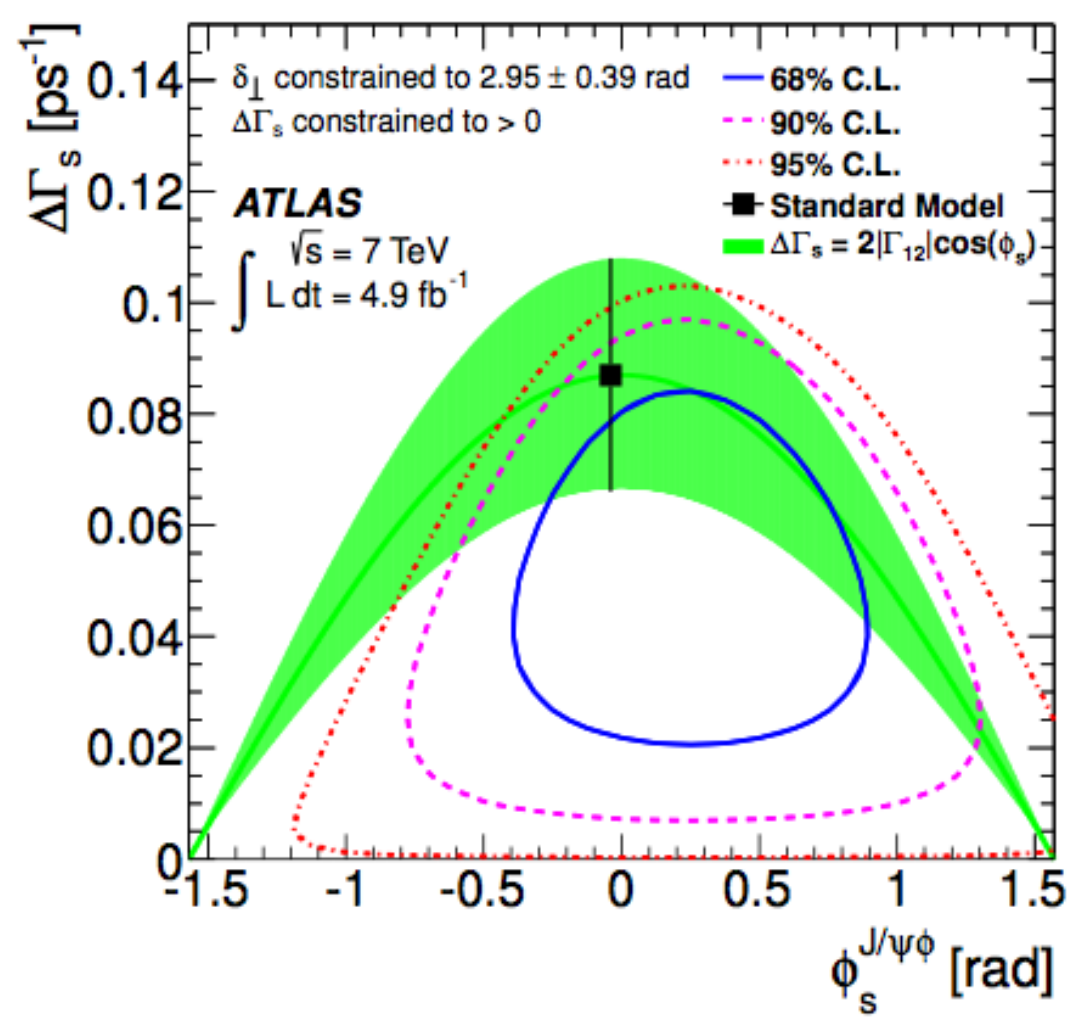

Figure 5: Likelihood contours in the $\phi_{s} \Delta \Gamma_{s}$ plane.The green band is the theoretical prediction of mixinginduced $C P$ violation. 
[5] ATLAS Collaboration, Observation of the $B_{c}^{ \pm}$in the decay $B_{c}^{ \pm} \rightarrow J / \psi \pi^{ \pm}$with the ATLAS detector at LHC, ATLAS Note ATLAS-CONF-2012-028

[6] K. Nakamura et al. J. Phys. G 37 (2010) 075021.

[7] ATLAS Collaboration, Measurement of the $\Lambda_{b}$ lifetime and mass in the ATLAS experiment, Physical Review D 87, 032002 (2013).

[8] R. Aaij et al. (LHCb Collaboration), Phys. Lett. B 708, 241 (2012).

[9] Aubert et al, Phys. Rev. Lett. 33, (1974) 1404 - 1406, doi:10.1103 Phys. Rev. Lett. 331404

[10] ATLAS Collaboration, First observation of the $J / \psi \mu^{+} \mu^{-}$resonance in ATLAS $p p$ collisions at $\sqrt{s}=7 \mathrm{TeV}$. ATLAS Note ATLAS-CONF-2010-045

[11] ATLAS Collaboration, Measurement of the differential cross-sections of inclusive, prompt and non-prompt $J / \psi$ production in $p p$ collisions at $\sqrt{s}=7 \mathrm{TeV}$. Nucl. Phys. B 850, 3, 21

[12] Cacciari M, Greco M and Nason P 2001 JHEP 0103006

[13] Barger V D, Keung W Y and Phillips R J N 1980 Phys. Lett. B 91253

[14] BargerVD, KeungWY and Phillips R J N 1980 Z.Phys. C6 169

[15] Lansberg J P 2010 Total J/ÏL production cross section at the LHC (Preprint hep-ph/1006.2750) Brodsky S J and Lansberg J P 2010 Phys. Rev. D 81 051502(R)

[16] Lansberg J P 2009 Eur. Phys. J. C 61693

[17] D.C. Hom, et al., Phys. Rev. Lett. 39 (1977)

[18] D.E. Acosta, et al., CDF Collaboration, Phys. Rev. Lett. 88 (2002) 161802, CDF

[19] Public Note 9966. [5] V.M. Abazov, et al., D0 Collaboration, Phys. Rev. Lett. 94 (2005) 232001;

[20] V.M. Abazov, et al., D0 Collaboration, Phys. Rev. Lett. 101 (2008) 182004.

[21] ATLAS Collaboration, Measurement of the $\Upsilon(1 S)$ production cross-section in pp collisions at $\sqrt{s}=7$ $\mathrm{TeV}$ in ATLAS, Physics Letters B 705 (2011)

[22] ATLAS Collaboration, Observation of a New $c h i_{b}$ State in Radiative Transitions to $\Upsilon(1 S)$ and $\Upsilon(2 S)$ at ATLAS, Phys. Rev. Lett. 108 (2012) 152001

[23] L. Moytka and K. Zaleski, Eur. Phys. J. C 4, 107 (1998).

[24] ATLAS Collaboration, Search for the decay $B_{s}^{0} \rightarrow \mu^{+} m u^{-}$with the ATLAS detector, Phys.Lett. B713 (2012) 387âĂŞ407. arXiv:1204.0735.

[25] R. Aaij, et al., Measurement of b hadron production fractions in 7 TeV pp collisions, Phys.Rev. D85 (2012) 032008. arXiv:1111.2357.

[26] A. L. Read, Presentation of search results: the CLs technique, Journal of Physics G: Nuclear and Particle Physics 28 (10) (2002) 2693.

[27] A. Hoecker, et al., TMVA 4, Toolkit for Multivariate Data Analysis with ROOT (2009). arXiv:physics/0703039.

[28] ATLAS Collaboration, ATL-COM-PHYS-2011-1619 (2011).

[29] ATLAS Collaboration, Search for the rare decays $B \rightarrow \mu \mu$ at the LHC with the ATLAS, CMS and LHCb experiments, ATLAS-CONF-2012-061 
[30] Time-dependent angular analysis of the decay $B_{s}^{0} \rightarrow J \psi \phi$ and extraction of $\Delta \Gamma_{s}$ and the $C P$-violating weak phase $\phi_{s}$ by ATLAS Phys. Rev. Lett. 108 (2012) 152001

[31] UTfit collaboration, M. Bona et al., Constraints on new physics from the quark mixing unitarity triangle, Phys. Rev. Lett. 97 (2006) 151803.

[32] LHCb collaboration, R. Aaij et al., Measurement of the $B_{s}-\bar{B}_{s}$ oscillation frequency $\Delta m_{s}$ in $B_{s}^{0} \rightarrow D_{s}(3) \pi$ decays, Phys. Lett. B 709 (2012) 177 [arXiv:1112.4311].

[33] A. Lenz and U. Nierste, Theoretical update of $B_{s} \bar{B}_{s}$ mixing, JHEP 06 (2007) 072 [hepph/0612167].

[34] CDF Collaboration, Phys. Rev. Lett. 107:272001(2011); D0 Collaboration, Phys. Rev. D 85,032006 (2012)

[35] LHCb Collaboration, CONF-2012-002 (2012)

[36] ATLAS collaboration, The ATLAS Experiment at the CERN Large Hadron Collider, 2008 JINST 3 S08003 